\title{
Adaptación y Validación de un Cuestionario de Compromiso Escolar en la Escuela Primaria
}

\section{Adaptation and Validation of a School Commitment Questionnaire in Primary School}

\author{
Rosario Gelpi Trudo 1,2, *, Macarena Verónica del Valle ${ }^{1,2}$, Carolina Magalí Pagano ${ }^{1}$, María Laura Andrés ${ }^{1,2}$, \\ Ana García Coni ${ }^{1,2}$ y Lorena Canet Juric ${ }^{1,2}$ \\ ${ }^{1}$ Universidad Nacional de Mar del Plata, Argentina \\ ${ }^{2}$ Consejo Nacional de Investigaciones Cientificas y Técnicas (CONICET), Argentina
}

\section{DESCRIPTORES:}

Compromiso escolar

Desafección

Adaptación

Psicometría

Educación

\begin{abstract}
RESUMEN:
El compromiso escolar es un constructo relacionado con la intensidad y calidad del involucramiento del estudiante en su aprendizaje y su escolaridad. Funciona como predictor de las trayectorias académicas y tiene sus inicios en la escuela primaria. Se ha propuesto un modelo de cuatro factores del compromiso escolar, que distingue entre compromiso y desafección, por un lado, y dimensión emocional y conductual, por otro. El compromiso escolar es un constructo complejo y relevante, pero su estructura ha sido poco explorada. Por lo tanto, el objetivo de esta investigación fue adaptar y presentar evidencias de las propiedades psicométricas de un cuestionario para el contexto argentino a través de la combinación en una única medida del Cuestionario para el alumno de Compromiso versus Desafección y algunos subdominios del Cuestionario para alumnos de Escuela Primaria. Para ello, se tradujeron y ajustaron los ítems de estos dos instrumentos y se administró a 269 estudiantes de segundo ciclo de educación primaria básica. Se realizó un análisis factorial exploratorio para conocer la estructura del instrumento. Se retuvieron 32 ítems, distribuidos en dos factores, con buenas evidencias de confiabilidad y validez de constructo y de criterio. La escala es válida para ser aplicada en el contexto argentino, con posibles impactos en la investigación y en la práctica psicoeducativa. Se discuten las implicaciones teóricas de la estructura obtenida.
\end{abstract}

\section{KEYWORDS:}

\section{ABSTRACT:}

School engagement

Disaffection

Adaptation

Psychometrics

Education
School engagement is a construct related to the intensity and quality of a student's involvement in his or her learning. The school engagement works as a predictor of academic performance and begins its development in primary education. A four-factor model of school engagement has been proposed, which distinguishes between engagement and disaffection, on the one hand, and emotional and behavioral dimensions, on the other. School engagement is a complex and relevant construct, but its structure has been scarcely explored. Thus, the aim of this study is to adapt a questionnaire to the Argentinian context and present its psychometric properties. The scale was developed combining the Engagement versus Disaffection with Learning and some sub-domains of the Student Self-report for Elementary School. The items of these two instruments were translated, adjusted and administered to 269 students (4th, 5th and 6th grade of primary education). An exploratory factor analysis was conducted to explore the structure of the instrument. The items were reduced to 32, and the analysis revealed a two-factor structure. The scale showed good evidence of reliability and validity. Therefore, the scale is valid to be applied in the Argentinean context, with possible impacts on research and psychoeducational practice. The theoretical implications of the structure obtained are discussed.

CÓMO CITAR:

Gelpi Trudo, R., del Valle, M. V., Pagano, C. M., Andrés, M. L., García Coni, A. y Canet-Juric, L. (2021). Adaptación y validación de un cuestionario de compromiso escolar en la escuela primaria. Revista Iberoamericana de Evaluación Educativa, 14(1), 173-189.

https://doi.org/10.15366/riee2021.14.1.010

*Contacto: rosariogelpitrudo@gmail.com

ISSN: 1989-0397

https://revistas.uam.es/riee 


\section{Introduccción}

El estudio del compromiso escolar (CE) se ha popularizado en las últimas décadas debido a su relación con el aprendizaje, la permanencia en el sistema educativo y el desempeño académico (Lei et al., 2018; Reschly y Christenson, 2012; Wonglorsaichon et al., 2014). El abordaje del CE brinda la posibilidad de mejorar las trayectorias educativas y de promover experiencias más positivas para los estudiantes, reduciendo el bajo desempeño, el desinterés y la deserción, especialmente en los sectores socioeconómicos más vulnerados (Finn y Zimmer, 2012). A pesar de su importancia, la estructura teórica del CE aún se encuentra en discusión (Tomas et al., 2016), y la diversidad de modelos para operacionalizarlo ha resultado en una gran heterogeneidad en las técnicas para su evaluación (Fredricks y McColksey, 2012; Sinatra et al., 2015). Por ello, el objetivo de este estudio fue desarrollar una medida del CE para ser aplicada en español y presentar evidencias de su validez y confiabilidad en población infantil argentina.

\subsection{Compromiso escolar}

Skinner y otros (2009) definen al CE como la intensidad y calidad del involucramiento del estudiante con las personas, actividades, tareas, metas, valores, costos y lugares que la escolaridad comprende. A pesar de que el estudio de CE es extenso, existe aún escaso consenso sobre su delimitación y estructura (Sinatra et al., 2015), lo que ha dado lugar a una multiplicidad de definiciones, en muchos casos contradictorias o ambiguas (Sandoval-Muñoz et al., 2018).

Algunos autores (p. ej., Fredricks et al., 2011; Lara et al., 2018; Sandoval-Muñoz et al., 2018) consideran que el CE puede abordarse desde una perspectiva interaccional, donde el CE sería el resultado de la interacción entre el niño (en desarrollo), sus tareas (cambiantes según las asignaturas y el momento) y el contexto (políticas y prácticas escolares e interacción con familia o pares; Sinclair et al., 2003; Skinner et al., 2009).

Uno de los modelos conceptuales del CE con mayor aceptación es el de Skinner et al. (2009), quienes proponen que el CE posee una estructura dual. Por un lado, se encuentra el CE propiamente dicho, compuesto por los procesos óptimos y adaptativos del funcionamiento escolar. Por el otro, como contraparte del CE, se encuentra la desafección, la cual abarca aquellos procesos disfuncionales y negativos. La desafección representa un factor en sí mismo que no se reduce a la mera ausencia de CE, sino que implica una participación escolar rutinaria y caracterizada por la sensación de obligatoriedad (Jang et al., 2016). A su vez, tanto el CE como la desafección se reflejan en conductas y en estados emocionales, determinando cuatro dimensiones en el modelo de Skinner et al. (2009): el compromiso emocional (estados afectivos relacionados con el involucramiento de los estudiantes durante las actividades de aprendizaje como entusiasmo, interés, disfrute, orgullo, vitalidad, ánimo), compromiso conductual (esfuerzo, la atención y persistencia al iniciar y ejecutar las tareas), desafección emocional (manifestación de emociones desvitalizantes, negativas y alienantes como aburrimiento, cansancio, frustración, ira, ansiedad, vergüenza o culpa), y desafección conductual (comportamientos que reflejan pasividad, falta de iniciativa y de esfuerzo).

Ahora bien, otros autores (p. ej., Fredricks et al., 2004; Reeve y Shin, 2020) han propuesto otras dimensiones para el CE. Por ejemplo, se ha propuesto una dimensión cognitiva, que referiría al uso de estrategias de aprendizaje, a la capacidad de autorregulación y al esfuerzo para comprender ideas complejas y dominar nuevas destrezas (Tomas et al., 2016). También se ha propuesto, una dimensión agéntica del $\mathrm{CE}$, según la cual los estudiantes contribuyen de manera constructiva a la instrucción que reciben estableciendo sus propias metas, tomando decisiones por sí solos y actuando de manera autónoma (Reeve y Shin, 2020). Mientras mayor es la participación activa de los estudiantes, mayor es la conceptualización que tienen sobre la calidad de su educación (Vilà et al., 2019). Sin embargo, estos componentes han sido discutidos debido a que en ocasiones se superponen con las dimensiones conductual y emocional, así como con otros constructos relacionados como motivación y autorregulación) (Sinatra et al., 2015).

La perspectiva de Skinner se incluye dentro de una teoría más amplia del desarrollo de la motivación: el Self-System Model of Motivational Development, basado en la teoría de la autodeterminación (SSMMD; Connell y Wellborn, 1991; Skinner et al., 2008). Este modelo enfatiza la importancia de la calidad de las interacciones sociales en el aula y plantea que el CE surge en la medida en que los alumnos perciban como satisfechas tres necesidades psicológicas básicas en el ámbito escolar (Wang y Hofkens, 2019). La prime- 
ra es la necesidad de competencia, que refiere a poder interactuar de manera efectiva con el ambiente escolar, conocer las estrategias necesarias para un buen desempeño, y ser capaz de ponerlas en práctica (Connell y Wellborn, 1991). En segundo lugar se encuentra la necesidad de autonomía, que implica sentir que se actúa por voluntad propia y expresando la propia individualidad (Furrer et al., 2014; Tian et al., 2016). Por último, la necesidad de relacionarse con los otros, refiere al sentimiento de pertenencia hacia la escuela y a la seguridad emocional en los vínculos con docentes, con pares y con uno mismo (Connell y Wellborn, 1991; Tian et al., 2016). Para que estas necesidades sean satisfechas, las relaciones con los docentes y con los pares deben caracterizarse por la inclusión, la confianza y la calidez (González-Alba et al., 2020; Furrer et al., 2014).

De esta manera, el grado percibido de satisfacción (o no) de esta tríada de necesidades predice las conductas y emociones que componen el CE y la desafección. Recientemente, estudios realizados en diversos contextos han brindado apoyo empírico a esta teoría. Por ejemplo, Wang et al. (2019) encontraron que la satisfacción de las necesidades básicas en la escuela se asociaba con el nivel de CE conductual y con el rendimiento académico. Por otro lado, Gutiérrez et al. (2018) reportaron que una gran parte de la varianza del CE se explica por la satisfacción de las necesidades psicológicas básicas.

\subsection{Impacto del CE sobre variables psicoeducativas en la escolaridad}

Diversos estudios (e.g., Fredricks et al., 2004; González et al., 2015; Miranda-Zapata et al., 2018; Rigo y Donolo, 2014) han demostrado que el CE se encuentra asociado al buen desempeño académico. El CE es también un factor promotor de otros resultados positivos como el mantenimiento del buen rendimiento a largo plazo, el interés por el aprendizaje, la asistencia a clases, las habilidades para la socialización, el bienestar del estudiante y la resiliencia académica (Miranda-Zapata et al., 2018; Tomás et al., 2016; Wang et al., 2019).

Aunque la mayor parte de las investigaciones se han enfocado en evaluar el CE en estudiantes secundarios y universitarios, resulta también relevante la indagación del CE durante la escolaridad primaria, ya que se trata de un período fundante del CE (las relaciones por fuera de la familia se incrementan, los niños comienzan a involucrarse en actividades extra-escolares y extra-curriculares, y la escolaridad se torna más formal; Mahatmya et al., 2012). Además, el grado de CE tiende a mantenerse entre la escolaridad primaria y la segundaria (Finn y Zimmer, 2012; Jang et al., 2016), por lo que, si existe un alto grado de desafección ya en este período inicial, el mismo se mantendrá y podría conducir al abandono de los estudios en la escolaridad secundaria. El estudio de esta problemática es particularmente valioso en Argentina, donde el abandono interanual pasa del 0,79\% en el nivel primario al 10,83\% en el nivel secundario (Ministerio de Educación y Deportes, 2017). Por esto, el nivel primario supone un momento clave para la prevención de estas dificultades a largo plazo y resulta necesario disponer de instrumentos de evaluación y trabajo adaptados a este período específico del desarrollo.

\subsection{Medición del CE y sus facilitadores}

La proliferación de estudios sobre el CE se ha visto acompañada por la construcción de una cantidad de instrumentos para su medición. Sin embargo, la diversidad en los modos de entender y operacionalizar el concepto ha resultado en una gran heterogeneidad en las técnicas de evaluación (Fernández da Lama, 2019; Fredricks y McColksey, 2012; Sinatra et al., 2015). Entre las medidas de CE se pueden mencionar las siguientes: Engagement Vs Disaffection with Learning (Skinner et al., 2008), Academic Engagement Scale for Grade School Students (Tinio, 2009), High School Survey of Student Engagement (Center for Evaluation and Educational Policy Indiana University, 2007), School Engagement Measure (Wang et al., 2011), Behavioral Engagement Questionnaire (Miserandino, 1996), Student Engagement in Mathematics Classroom Scale (Kong et al., 2003), Motivation and Engagement Scale (Martin, 2009), Student Engagement in School-Four-Dimensional Scale (Veiga, 2013), y Student Engagement Instrument (Appleton et al., 2006). Sin embargo, como puede observarse, la mayoría de estas técnicas se encuentran en inglés y ninguna ha sido adaptada en Argentina. Dentro del contexto latinoamericano, Lara y otros (2018) han desarrollado un cuestionario destinado a adolescentes, validado en población chilena, y Peña et al. (2016) validaron la Escala de Involucramiento Académico en estudiantes universitarios de Venezuela. 
A partir de su modelo teórico, Skinner y otros (2008) construyeron el Cuestionario de Compromiso versus Desafección (CC/D; Engagement versus Disaffection with Learning: Student-Report), el cual evalúa el nivel de CE y desafección a través de un auto-reporte para la escolaridad primaria. En niños estadounidenses de 7 a 13 años la escala mostró una estructura de cuatro factores con buen ajuste a los datos (CFI = ,98; RMSEA = ,08), buenos índices de confiabilidad ( $\alpha$ entre ,65 y ,82) y evidencias de validez convergente (correlaciones con percepción del control en el aula, orientación a metas, percepción de apoyo social, percepción de autonomía, optimismo/pesimismo académico y reacción ante el desafío; Skinner et al., 2009). En un estudio posterior, Immekus y Ingle (2019) realizaron un Análisis Factorial Exploratorio (AFE) de la escala, observando, contrariamente a los autores originales, una estructura de tres factores (desafección emocional, compromiso emocional y compromiso conductual), con un ajuste aceptable a los datos (CFI = ,95; RMSEA = ,07) y adecuados niveles de confiabilidad (coeficiente omega entre ,75 y ,81).

Basándose en el mismo modelo teórico, el Institute for Research and Reform in Education (1998) diseñó el Cuestionario para Alumnos de Escuela Primaria (CAEP; Research Assessment Package For Schools: Student Self-report for Elementary School) para medir diversos procesos psicológicos e interpersonales que impactan sobre el desempeño y el ajuste de los estudiantes en la escuela. Entre ellos, el dominio Creencias sobre sí mismo evalúa las percepciones de satisfacción de las necesidades de competencia, autonomía y relación con los otros (que, como se explicó previamente, se reconocen como facilitadoras del CE). Las propiedades psicométricas del CAEP fueron analizadas en estudiantes estadounidenses de escuelas primarias, mostrando niveles de consistencia interna entre bajos y adecuados ( $\alpha$ entre ,52 y ,87 para los distintos subdominios, y , 87 para el dominio total) y evidencias de validez mediante asociaciones con criterios externos (compromiso del estudiante, reportes docentes e índices de rendimiento escolar). Empero, los autores no presentan evidencias sobre la validez de constructo de la escala.

Tanto el CC/D como el CAEP carecen de adaptaciones previas al español, y es aún escasa la evidencia sobre el funcionamiento psicométrico de ambos. Además, respecto de la adaptación de instrumentos, las propiedades psicométricas de un test no pueden considerarse a priori, sino que deben demostrarse empíricamente, pudiendo estas variar al aplicar la medida en una cultura diferente a la de origen (Chahín-Pinzón, 2014), o pudiendo variar incluso la forma en que la variable a medir se manifiesta según la cultura (Molinari y Sánchez Rosas, 2018). Por ello, y dado que no se registra ningún instrumento de evaluación de estas características que haya sido adaptado para el contexto argentino y para la escolaridad primaria, el objetivo de esta investigación fue combinar el CC/D y el CAEP en una medida única, adaptarla al español y presentar evidencias de su validez y confiabilidad en población infantil argentina. La versión final del cuestionario fue llamada Cuestionario de Dimensiones y Facilitadores del Compromiso Escolar.

\section{Método}

\subsection{Enfoque metodológico}

El estudio fue de carácter instrumental, con un enfoque cuantitativo destinado al desarrollo de una prueba de evaluación (Montero y León, 2002). Se espera lograr que la prueba adaptada sea válida y confiable para su uso en el habla hispana y equivalente en su funcionamiento conceptual, de contenido, técnico y de criterio con otros cuestionarios utilizados en otros países (Carvajal et al., 2011). La elaboración del nuevo cuestionario se llevó a cabo en etapas: trabajo de traducción y adaptación de ítems, redacción de la versión inicial del cuestionario en español, revisión por jueces expertos, pilotaje y validación de la versión final.

\subsection{Variables}

La variable principal bajo estudio fue el CE de los niños 9 y 12 años de edad. También se trabajó con variables de criterio para evaluar la validez de la escala, a saber, las habilidades académicas de cálculo y comprensión lectora y las calificaciones escolares reportadas por los docentes. 


\subsection{Instrumentos}

Para la selección de los instrumentos, se consideró pertinente la utilización medida de autoinformes, dada su adecuación para la evaluación de estados internos, así como sus ventajas en costo, tiempo de administración y confiabilidad con respecto a otros tipos de técnicas (Duckworth y Yeager, 2015). Se buscaron instrumentos específicamente diseñados para el nivel primario, dado que otras etapas del desarrollo reflejan distintas habilidades, conductas y emociones asociadas al CE (Sinatra et al., 2015). Además, se buscó que los cuestionarios evaluaran tanto el estado actual del CE/desafección del estudiante, como los factores interpersonales que subyacen a su desarrollo y sostenimiento. Dado que no se encontró ningún instrumento que, por sí solo, aportara una evaluación tan completa, se tomó la decisión de combinar dos instrumentos ampliamente utilizados. La combinación de instrumentos es una práctica común para la mejora de las propiedades psicométricas de los instrumentos de evaluación (p. ej., McHugh y Otto, 2012). Finalmente, los dos instrumentos escogidos lograban la complementariedad buscada, siendo factible su unión gracias a que comparten una misma base teórica (Bolio Domínguez y Pinzón Lizarraga, 2019).

- Compromiso escolar/desafección: Cuestionario de Compromiso versus Desafección (CC/D; Skinner et al, 2008). Este cuestionario evalúa las variables de compromiso hacia el aprendizaje escolar, y está dirigido a estudiantes entre $4^{\circ}$ y $7^{\circ}$ año de su escolaridad ( 9 a 12 años). Se compone de 20 ítems, divididos en cuatro subescalas: Compromiso Conductual, Compromiso Emocional, Desafección Conductual y Desafección Emocional. Esta última dimensión incluye siete ítems suplementarios que indagan emociones negativas específicas (frustración, ansiedad y aburrimiento) propias del contexto escolar. Los estudiantes responden según una escala de tipo Likert, desde 1 (para nada verdadero) hasta 4 (muy verdadero).

- Facilitadores del CE/desafección: Dominio Creencias sobre sí mismo del Cuestionario para alumnos de Escuela Primaria (CAEP, Institute for Research and Reform in Education, 1998). Del mismo, se seleccionaron cinco subdominios: Creencias promotoras de la competencia (6 ítems), Creencias obstaculizadoras de la competencia (10 ítems), Autorregulación intrínseca (4 ítems), Autorregulación por identificación (5 ítems) y Seguridad emocional respecto a los docentes ( 3 ítems). Las creencias promotoras y obstaculizadoras de la competencia refieren a las percepciones del alumno sobre su control del ambiente escolar y sobre las estrategias que cree necesarias para su buen desempeño (p. ej., "No sé qué tengo que hacer para sacarme buenas notas"). La autorregulación intrínseca y la autorregulación por identificación indagan sobre la motivación del alumno a realizar las tareas (e.g., "Hago la tarea en casa porque me gusta hacerla"). Por último, la seguridad emocional respecto a los docentes evalúa la percepción de los vínculos del alumno con sus maestros (p. ej., "Siento que mi maestro me ignora"). Este instrumento se responde según una escala Likert de cuatro puntos, que incluye tanto ítems de valor negativo como positivo.

- Comprensión lectora: Test Leer para Comprender (TLC, Abusamra et al., 2010). Se compone de dos pruebas diseñadas para evaluar comprensión de textos narrativos y expositivos. El alumno debe contestar 13 y 12 preguntas, respectivamente, sobre cada texto leído, marcando la respuesta correcta entre cuatro alternativas. El tiempo promedio de administración es 20 minutos. Se ha reportado un nivel de confiabilidad aceptable tanto para niños de $4^{\circ}$ como de $5^{\circ}$ año de escuela primaria ( $\alpha$ desde ,66 hasta ,71) (Abusamra et al., 2010).

- Cálculo aritmético: Subtest de Aritmética de la Prueba de Logro de Amplio Rango WRAT-3 (Wilkinson, 1993). Evalúa las habilidades básicas de conteo, lectura de símbolos numéricos, resolución de problemas orales y cálculos escritos. Los ítems aumentan de dificultad, desde problemas de adición de un dígito hasta problemas de álgebra que contienen varias variables. El tiempo de administración es de 15 minutos. La prueba ha mostrado previamente adecuados niveles confiabilidad en el contexto argentino (Andrés et al., 2017; Vernucci et al., 2017).

- Calificaciones escolares: Fueron recolectadas a partir de los informes del tercer trimestre y finales del ciclo lectivo 2019, en las asignaturas Matemática y Prácticas del Lenguaje. 


\subsection{Participantes, procedimiento y consideraciones éticas}

El presente estudio fue aprobado por la Agencia de Promoción Científica y Tecnológica de Argentina (1614/2017). En primer lugar, se realizó una traducción y adaptación de los ítems de los cuestionarios CC/D y CAEP. Se redactó una versión inicial del cuestionario en español, cuidando conservar la equivalencia conceptual. Se combinaron ambas traducciones en un único cuestionario, con una misma escala de respuesta (tipo Likert de cuatro opciones, según el grado de acuerdo con el ítem), y se aleatorizó el orden de los ítems.

En segundo lugar, el cuestionario fue revisado por cuatro jueces expertos. A partir de sus sugerencias, y con el fin de aumentar la validez de contenido del instrumento (Bolio Domínguez y Pinzón Lizarraga, 2019), se decidió remover cinco ítems pertenecientes al dominio "Creencias obstaculizadoras de la competencia" del CAEP, por ser estos redundantes y porque su enunciación como negaciones podría resultar confusa para los niños a la hora de responder ("No sé cómo dejar de sacarme malas notas"; "Si no tengo suerte, no me va a ir bien en la escuela"; "No puedo lograr caerle bien a mi maestro/a"; "No puedo esforzarme mucho en la escuela"; "No soy inteligente en la escuela").

Posteriormente, se tomó una prueba piloto a una muestra pequeña de niños $(n=6)$, en la que se les solicitó que respondieran el cuestionario y marcaran aquellos ítems que les hubieran resultado de difícil comprensión, y se los invitó a que realizaran sugerencias. Esto permitió hacer ajustes en los ítems para que sean más coloquiales y comprensibles, adecuándolos a la población seleccionada. Finalmente, se confeccionó la versión final del cuestionario fue administrada para su validación.

Para este estudio, se trabajó con dos escuelas primarias de la ciudad de Mar del Plata, provincia de Buenos Aires, Argentina, las cuales fueron seleccionadas a través de un acuerdo con la Jefatura distrital de Inspección General de Educación de la Provincia de Buenos Aires. Las instituciones educativas fueron contactadas e invitadas a participar del estudio. Luego, se realizaron reuniones con padres/tutores y docentes para explicar la naturaleza del estudio y se entregaron hojas de información. Se proporcionó también a los niños la información necesaria para la comprensión de los objetivos del estudio, su propósito y procedimientos a implementar. Aquellos padres/tutores que accedieron voluntariamente a participar, firmaron un consentimiento informado. Los niños también debían dar su asentimiento, tanto de manera escrita como oral. Durante el estudio se garantizó la seguridad y comodidad de los niños, y se les recordó que tenían la posibilidad de revocar en cualquier momento su decisión de participar. La confidencialidad de la información recabada fue resguardada en todas las instancias de la investigación. De esta manera, se respetaron los lineamientos éticos sugeridos por entidades nacionales e internacionales (APA, 2010; CONICET, 2006; WMA, 2013).

Se evaluó a un total de 269 niños de ambos sexos (femenino: 53,9\%, $\mathrm{n}=145$; masculino; $46,1 \%, \mathrm{n}=$ $124)$, con un rango de edad entre 9 y 12 años $(\mathrm{ME}=10,47 ; \mathrm{DE}=1,37)$, concurrentes a $4^{\circ}(27,9 \%, \mathrm{n}=$ $75), 5^{\circ}(36,8 \%, \mathrm{n}=99)$ y $6^{\circ}(35,3 \%, \mathrm{n}=95)$ año de Escolaridad Primaria Básica en dos instituciones educativas de gestión pública de la ciudad de Mar del Plata. La selección muestral se realizó de forma no probabilística intencional en función de los siguientes criterios: que los alumnos incluidos en la muestra no presentasen antecedentes de trastornos del aprendizaje, del desarrollo o psicopatológicos, antecedentes psiquiátricos ni de disfunción neurológica, y no tomasen medicación psicotrópica.

Los cuestionarios fueron administrados de forma individual por evaluadores capacitados. La evaluación se realizó durante el horario escolar, en una sala de la escuela destinada y acondicionada para tal fin. Las pruebas de habilidades académicas se tomaron de manera grupal en el aula, en horarios de las asignaturas correspondientes (Matemática y Prácticas del Lenguaje) cedidas a tal fin por parte de los docentes. Las calificaciones escolares fueron solicitadas a los docentes de cada curso.

\subsection{Análisis de datos}

Los ítems inversos fueron recodificados para calcular una variable total con la sumatoria de los 55 ítems del cuestionario. Para evaluar el nivel de asociación entre cada ítem y la escala total (ítem-total correlation, Wieland et al., 2017), se aplicaron correlaciones $r$ de Pearson. Mediante este procedimiento se observó al grado de relación entre cada ítem con el puntaje total y se eliminaron los ítems con correlaciones nulas. 
Con la totalidad de los ítems sin recodificar, se evaluó la aplicabilidad del AFE a través de la prueba de esfericidad de Bartlett y el estadístico Kaiser-Meyer-Olkin. El AFE es una técnica que permite explorar las dimensiones subyacentes o variables latentes de un grupo de variable observadas (los ítems en este caso). El AFE se realizó por medio del programa FACTOR (v. 10.10.01, Lorenzo-Seva y Ferrando, 2019). Primero se determinó el número de factores a extraer a través de un análisis paralelo con implementación óptima (Timmerman y Lorenzo-Seva, 2011) en base a la matriz de correlaciones policóricas. Se optó por no trabajar con el procedimiento de extracción de Kaiser de autovalores mayores a 1, puesto que este método tiende a sobreestimar la cantidad de factores a extraer ( $\mathrm{Fe}$ rrando y Anguiano-Carrasco, 2010). El método de extracción utilizado fue el de mínimos cuadrados no ponderados (ULS), robusto frente a variables ordinales (Lloret-Segura et al., 2014). La rotación posteriormente aplicada fue la oblicua Promax, considerada para cuando se asumen correlaciones interfactoriales (Henson y Roberts, 2006). Se conservaron solo los ítems cuyas cargas factoriales fueran mayores a los ,40 puntos y cuyas saturaciones en los dos primeros factores presentaran una diferencia mayor a ,30 puntos, siguiendo recomendaciones previas (Lloret-Segura et al., 2014; Wieland et al., 2017). Con los ítems conservados se realizó un segundo y un tercer AFE bajo los mismos métodos utilizados para el primero.

La consistencia interna de los factores fue evaluada mediante el índice alfa de Cronbach ( $\alpha)$. La correlación interfactorial se calculó mediante el índice $r$ de Pearson. Finalmente, para indagar la validez de criterio, se calcularon correlaciones $r$ de Pearson entre las puntuaciones del instrumento e indicadores de habilidades académicas y rendimiento escolar. A excepción del AFE, todos los análisis fueron realizados con el programa SPSS v.25.

\section{Resultados}

A continuación, se presentan los resultados del estudio. En primer lugar, se describirá la estructura factorial del instrumento. Luego, se reportarán los índices de consistencia interna de cada factor y del instrumento completo. Finalmente, se brindarán los resultados del análisis de la validez de criterio del instrumento, en función de sus correlaciones con el desempeño académico.

\subsection{Exploración de la estructura factorial}

En primer lugar, se analizó la correlación de los 55 ítems con la variable total del cuestionario de CE, lo que reveló la necesidad de eliminar dos ítems ("Tengo suerte en las evaluaciones" y "Puedo conseguir caerle bien a mi maestro o maestra") por no presentar correlaciones estadísticamente significativas. Luego, se corroboró la aplicabilidad del AFE con resultados satisfactorios (KMO = ,93; Bartlett (1128) $=2891,7 ; p<, 01)$. El AFE sugirió retener dos factores, que explicaban el $41,52 \%$ de la varianza. Con base en los criterios antes mencionados, se conservaron 34 ítems con cargas factoriales mayores a los , 40 puntos y cuyas saturaciones en los dos primeros factores presentaran una diferencia mayor a ,30 puntos. Con los 34 ítems se calculó un segundo AFE que confirmó la existencia de dos factores que explicaban el $48,23 \%$ de la varianza. Al observar la matriz rotada, y en base a los mismos criterios, se eliminaron otros dos ítems. El tercer y último AFE realizado sobre los 32 ítems finales reveló una estructura de dos factores, con una varianza explicada de 50,42\%. El primer factor $(35,43 \%$ de la varianza) estuvo compuesto por 19 ítems y fue llamado "Desafección hacia la escolaridad y obstaculizadores del CE" $(\alpha=, 90)$. El segundo factor $(14,99 \%$ de la varianza) estuvo compuesto por un total de 13 ítems y fue llamado "Compromiso escolar y facilitadores" $(\alpha=, 84)$. Las cargas factoriales de la versión final de 32 ítems de la escala se presentan en el Cuadro 1. Las correlaciones inter-factoriales fueron de $r=-, 32(p<, 01)$. El instrumento completo presentó un índice alto de consistencia interna $(\alpha=, 89)$. 
Cuadro 1

Cargas factoriales del Cuestionario de Dimensiones y Facilitadores del Compromiso Escolar

\begin{tabular}{|c|c|c|}
\hline & \multicolumn{2}{|c|}{ Factores } \\
\hline & 1 & 2 \\
\hline 1. Me esfuerzo para que me vaya bien en la escuela. & & ,73 \\
\hline 2. Cuando estoy en clase, me siento bien. & &, 51 \\
\hline 6. Disfruto de aprender cosas nuevas en clase & & ,74 \\
\hline 10. Me interesan las cosas que hacemos en clase & & ,73 \\
\hline 13. En clase, pongo todo mi esfuerzo & & ,70 \\
\hline 16. Cuando estoy en clase, escucho atentamente & & ,65 \\
\hline 18. Me engancho con las actividades que hacemos en clase & & ,67 \\
\hline 26. Estar en clase es divertido & & ,64 \\
\hline 30. Hago la tarea en casa porque quiero entender el tema & & ,57 \\
\hline 34. Trabajo en las actividades de clase porque me resultan interesantes & & ,78 \\
\hline 37. Pienso que es importante hacer las actividades de clase & & ,70 \\
\hline 39. Hago las actividades de clase porque quiero aprender cosas nuevas & & 87 \\
\hline 41. Esforzarme es el mejor modo de que me vaya bien en la escuela & & ,67 \\
\hline 43. Hago las actividades de clase porque es divertido & & ,78 \\
\hline 47. Hago la tarea en casa porque me gusta hacerla & & ,67 \\
\hline $\begin{array}{l}\text { 49. Hago las actividades de clase porque me importa que me vaya bien en } \\
\text { la escuela }\end{array}$ & & ,74 \\
\hline 55. Hago la tarea en casa porque quiero aprender cosas nuevas & & ,71 \\
\hline 57. Puedo esforzarme mucho en la escuela & & ,67 \\
\hline 59. Hago la tarea en casa porque es divertido & & ,67 \\
\hline 14. Me siento mal cuando estoy en clase & ,46 & \\
\hline 17. Me aburro cuando mi maestro o maestra explica algo nuevo &, 59 & \\
\hline 21. En la clase hago lo mínimo, como para zafar & ,62 & \\
\hline 25. Me siento enojado cuando tengo que hacer actividades en clase &, 58 & \\
\hline 28. No puedo hacer que me vaya bien en la escuela & ,64 & \\
\hline $\begin{array}{l}\text { 29. El mejor modo de sacarme buenas notas es caerle bien a mi maestro o } \\
\text { maestra }\end{array}$ & ,73 & \\
\hline 40. Siento que mi maestro o maestra me ignora &, 55 & \\
\hline 42. No me va a ir bien en la escuela si a mi maestro o maestra le caigo mal & ,62 & \\
\hline 45. Tengo que tener suerte para que me vaya bien en la escuela & ,75 & \\
\hline 48. Me siento enojado cuando estoy con mi maestro o maestra & ,68 & \\
\hline 51. No sé qué tengo que hacer para sacarme buenas notas & ,78 & \\
\hline 58. No me siento feliz cuando estoy con mi maestro o maestra & ,62 & \\
\hline 61. No tengo suerte en la escuela & ,60 & \\
\hline
\end{tabular}

Nota. Elaboración propia. 


\subsection{Validez de criterio}

Se evaluó la validez del instrumento a partir de su relación criterios externos, a saber, calificaciones escolares, pruebas de rendimiento en comprensión lectora y cálculo aritmético. Los resultados de las correlaciones $r$ de Pearson y los valores estadística descriptivos se presentan en el Cuadro 2.

Cuadro 2

Análisis de validez de criterio del Cuestionario de Dimensiones y Facilitadores del Compromiso Escolar

\begin{tabular}{lccccccc}
\hline & $\mathbf{1}$ & $\mathbf{2}$ & $\mathbf{3}$ & $\mathbf{4}$ & $\mathbf{5}$ & $\mathbf{M E}$ & $\mathbf{D E}$ \\
\cline { 2 - 7 } $\begin{array}{l}\text { 1. Compromiso escolar y } \\
\text { facilitadores }\end{array}$ & - & - & - & - & - & 3,43 & 0,49 \\
$\begin{array}{l}\text { 2. Desafección y } \\
\text { obstaculizadores }\end{array}$ &,$- 32^{* *}$ & - & - & - & - & 0,65 & 0,56 \\
3. TLC &,- 06 &,$- 46^{* *}$ & - & - & - & 34,83 & 8,87 \\
4. WRAT &, $16^{*}$ &,$- 30^{* *}$ &, $45^{* *}$ & - & - & 13,44 & 3,26 \\
5. Calificación lengua &, $21^{* *}$ &,$- 50^{* *}$ &, $50^{* *}$ &, $58^{* *}$ & - & 7,88 & 1,09 \\
6. Calificación matemática &, $20^{* *}$ &,$- 52^{* *}$ &, $38^{* *}$ &, $55^{* *}$ &, $84^{* *}$ & 7,77 & 1,06 \\
\hline
\end{tabular}

Notas: ${ }^{*} p<, 05 ;{ }^{* *} p<, 01$.

Los resultados muestran que existen relaciones significativas moderadas entre las dimensiones de la escala y los criterios externos. Mientras mayores son los niveles de CE, mejor es el desempeño académico y mejor el rendimiento en tareas de comprensión lectora y cálculo matemático. Sin embargo, el factor Compromiso no se asocia de manera significativa con el puntaje en la prueba de comprensión lectora TLC. Las correlaciones fueron más fuertes para la dimensión Desafección que para la dimensión Compromiso.

\section{Discusión}

Este trabajo tuvo como objetivo general la construcción de un instrumento de evaluación del CE a partir de la traducción, adaptación y validación de dos medidas previamente existentes. A partir de los análisis, del total de 55 ítems originales se retuvieron solo 32. La depuración de las escalas es una práctica usual en estudios psicométricos (Wieland et al., 2017) puesto que permite la mejora de las propiedades de los instrumentos. Se optó por criterios rigurosos en la conservación de ítems, permitiendo una medida abreviada, con mayor utilidad práctica, más sencilla de interpretar y rápida de aplicar (Reich et al., 2020). Los resultados sugirieron la existencia de dos factores. El factor “Compromiso escolar y facilitadores”, agrupó ítems relacionados con el compromiso emocional y conductual del alumno, así como distintos facilitadores del CE (percepción de la propia competencia, autorregulación del aprendizaje, motivación intrínseca). El factor "Desafección hacia el aprendizaje y obstaculizadores del CE", incluyó ítems relativos a la desafección conductual y emocional, y a los obstaculizadores del CE como las creencias disfuncionales sobre las propias capacidades y la insatisfacción emocional en los vínculos con los maestros.

Similar a lo propuesto por Skinner y otros $(2008,2009)$, los resultados respaldan que el CE y la desafección serían componentes diferenciables. Pero, a diferencia de lo indicado por estos autores y por otros estudios (p. ej., Immekus e Ingle, 2019), el análisis no mostró una distinción entre los componentes emocionales y conductuales. Tampoco se halló evidencia de otras dimensiones sugeridas por algunos autores, como la cognitiva (Tomas et al., 2016) o la agéntica (Reeve y Shin, 2020). El apoyo de los docentes a la autonomía del estudiante, la autoeficacia, los vínculos adaptativos, y demás facilitadores, parecen aglutinarse en una única dimensión asociada al CE. Por el contrario, los pensamientos descalificadores, las relaciones disfuncionales o negativas, y demás obstaculizadores se unifican en un único componente de desafección (Jang et al., 2016). No obstante, aún son necesarios estudios ulteriores que confirmen esta estructura bidimensional por medio de Análisis Factoriales Confirmatorios.

Respecto a las propiedades psicométricas del instrumento, el mismo mostró un alto nivel de consistencia interna y evidencias de validez de criterio con las calificaciones escolares y tareas de habilidades acadé- 
micas (cálculo matemático y comprensión lectora). Las correlaciones fueron significativas, moderadas, y positivas, similares a los reportado por estudios previos, respecto de la relación entre el CE y el desempeño escolar (Lei et al., 2018; Wonglorsaichon et al., 2014). Cabe destacar que el CE se vio más fuertemente relacionado con las calificaciones escolares que con el desempeño en las habilidades académicas. Esto puede deberse a que el CE también refiere a otros factores vinculados con la adaptación a la escuela, y que inciden en las notas escolares, tales como la participación en clase, la adhesión a normas de conducta, la relación con cada docente y el compañerismo (Griffiths et al., 2009). Además, debe notarse que la dimensión "Compromiso Escolar y Facilitadores" no se asoció con la comprensión lectora. Estudios previos, también han reportado hallazgos similares respecto de la relación entre distintos procesos psicológicos y la comprensión lectora y el cálculo matemático. Por ejemplo, Zamora et al. (2020) hallaron que el reporte paterno de los niveles de impulsividad de los niños se asociaba con el cálculo matemático, pero no con la comprensión lectora, y del Valle et al. (2019) indican que mientras la relación entre el uso de estrategias desadaptativas de regulación emocional y el cálculo matemático es moderada $(-, 47)$, la relación con la comprensión lectora es significativamente inferior $(-, 26)$. También, debe tenerse en cuenta que la comprensión lectora es un proceso complejo integrado por múltiples niveles, y que la medida utilizada puede no haber sido lo suficientemente exhaustiva en la indagación del rendimiento en esta área.

\section{Conclusiones}

Este estudio ha brindado una primera versión de un instrumento sencillo, válido y confiable para la evaluación del CE y sus facilitadores en estudiantes de segundo ciclo de escuela primaria, adaptado a las pautas lingüísticas, conceptuales y culturales del contexto argentino. Por lo tanto, el mismo podrá ser una herramienta útil como base para la implementación de intervenciones en el aula, que tiendan a conocer e incrementar los niveles de CE para la mejora del desempeño, así como para la potenciación de otros efectos positivos (por ejemplo, el aumento del bienestar de los estudiantes, o la generación de resiliencia académica) y la prevención de problemáticas escolares a corto y a largo plazo (deserción, desinterés). Asimismo, el cuestionario supone una herramienta en el ámbito de la investigación, en aquellos estudios en que esté implicado el CE.

Es preciso señalar ciertas limitaciones. Se debe tener en cuenta que corresponde a una primera exploración del instrumento, siendo pequeño (aunque adecuado) el tamaño de la muestra (Kyriazos, 2018), y que esta presenta cierta homogeneidad en cuanto a las características sociodemográficas de sus integrantes, pertenecientes a dos colegios de gestión pública cuyas poblaciones son similares en cuanto a nivel socioeconómico y grupo cultural de pertenencia. Esto limita parcialmente la posibilidad de generalización de estos resultados, reduciendo el alcance poblacional de la adaptación realizada. Además, sería aconsejable que se llevase a cabo la repetición del proceso mediante una validación cruzada, debido a las fluctuaciones que los estadísticos derivados de las puntuaciones de los ítems pueden presentar en función de la muestra utilizada (Carretero-Dios y Pérez, 2005). Por ello, este trabajo deja abierta la posibilidad de seguir trabajando sobre este instrumento en estudios futuros, que tengan el propósito de ampliar el alcance de este cuestionario, adaptándolo a otros rangos etarios y estratos sociodemográficos. Por otra parte, es necesario realizar estudios confirmatorios sobre la estructura encontrada. Finalmente, el CE es un componente aún poco analizado, pero el estudio de las variables que afectan a la motivación y previenen el fracaso no debe limitarse solo a él. El CE se asocia con otra multiplicidad de fenómenos que aún es necesario explorar mediante nuevos estudios para poder tener una mayor comprensión de los procesos educativos e intervenir en formas más efectivas.

\section{Referencias}

Abusamra, V., Ferreres, A., Raiter, A., De Beni, R. y Cornoldi, C. (2010). Test leer para comprender TLC. Evaluación de la comprensión de textos. Paidós.

American Psychological Association (2010). Ethical principles of psychologists and code of conduct. http://www.apa.org/ethics/code/principles.pdf

Andrés, M. L., Stelzer, F., Canet Juric, L., Introzzi, I., Rodriguez-Carvajal, R. y Navarro Guzmán, J. I. (2017). Emotion regulation and academic performance: a systematic review of empirical relationships. Psicologia em Estudo, 
22(3), 299-311.

https://doi.org/10.4025/psicolestud.v22i3.34360

Appleton, J., Christenson, S., Kim, D. y Reschly, A. (2006). Measuring cognitive and psychological engagement: Validation of the student engagement instrument. Journal of School Psychology, 44(5), 427-445.

https://doi.org/10.1016/j.jsp.2006.04.002

Bolio Domínguez, V. y Pinzón Lizarraga, L. M. (2019). Construcción y validación de un instrumento para evaluar las características de la responsabilidad social universitaria en estudiantes universitarios. Revista Internacional De Educación para la Justicia Social, 8(1), 79-96.

https://doi.org/10.15366/riejs2019.8.1.005

Carretero-Dios, H. y Pérez, C. (2005). Normas para el desarrollo y revisión de estudios instrumentales. International Journal of Clinical and Health Psychology, 5(3), 521-551.

Carvajal, A., Centeno, C., Watson, R., Martínez, M. y Sanz Rubiales, A. (2011). ¿Cómo validar un instrumento de medida de la salud? Anales del Sistema Sanitario de Navarra, 34(1), 63-72.

https://doi.org/10.4321/s1137-66272011000100007

Center for Evaluation y Education Policy. (2007). Voices of students on engagement: A report on the 2006 High School Survey of Student Engagement. https://files.eric.ed.gov/fulltext/ED495758.pdf

Chahín-Pinzón, N. (2014). Aspectos a tener en cuenta cuando se realiza una adaptación de test entre diferentes culturas. Psychologia: Avances de la Disciplina, 8(2), 109-112.

https://doi.org/10.21500/19002386.1225

Clarkson, D. B. y Jennrich, R. I. (1988). Quartic rotation criteria algorithms. Psychometrika, 53(2), 251-259. https://doi.org/10.1007/BF02294136

Connell, J. P. y Wellborn, J. G. (1991). Competence, autonomy, and relatedness: A motivational analysis of self-system processes. En M. R. Gunna, y L. A. Sroufe (Eds.), The Minnesota symposia on child psychology (Vol. 23). Self -processes and development (pp. 43-77). Lawrence Erlbaum Associates.

Consejo Nacional de Investigaciones Científicas y Técnicas. (2006). Lineamientos para el comportamiento ético en las ciencias sociales y humanidades $(\mathrm{CS} y \mathrm{H})$. Ministerio de Educación, Ciencia y Tecnología de Argentina.

del Valle, M., Zamora, E. V., Irurtia, M. J., Andrés, M. L. y Urquijo, S. (2019). Efecto del uso de estrategias cognitivas de regulación emocional sobre las habilidades académicas de cálculo y competencia lectora en adolescentes. Investigaciones en Psicología, 24(1), 5-23.

Duckworth, A. L. y Yeager, D. S. (2015). Cognitive ability for educational purposes. Educational Researcher, 44(4), 237-251. https://doi.org/10.3102/0013189X15584327.Measurement

Fernández da Lama, R. (2019). Student engagement: a non-systematic review of its conceptualization, models and assessment instruments. Investigaciones en Psicología, 24(1), 87-95.

Ferrando, P. J. y Anguiano-Carrasco, C. (2010). El análisis factorial como técnica de investigación en Psicología. Papeles del Psicólogo, 31(1), 18-33.

Finn, J. D. y Zimmer, K. S. (2012). Student engagement: What is it? Why does it matter? En S. L. Christenson, A. L. Reschly y C. Wylie (Ed.), Handbook of research on student engagement (pp. 97-132). Springer. https://doi.org/10.1007/978-1-4614-2018-7_5

Fredricks, J. A., Blumenfeld, P. C. y Paris, A. H. (2004). School engagement: Potential of the concept, state of the evidence. Review of Educational Research, 74(1), 59-109.

https://doi.org/10.3102/00346543074001059

Fredricks, J. A. y McColskey, W. (2012). The measurement of student engagement: A comparative analysis of various methods and student self-report instruments. En S. L. Christenson, A. L. Reschly y C. Wylie (Ed.), Handbook of Research on Student Engagement (pp. 763-782). Springer. https://doi.org/10.1007/978-1-4614-2018-7_5

Fredricks, J., McColskey, W., Meli, J., Mordica, J., Montrosse, B. y Mooney, K. (2011). Measuring student engagement in upper elementary through high school: A description of 21 instruments. Issues \& Answers, 98.

https://ies.ed.gov/ncee/edlabs/regions/southeast/pdf/rel_2011098.pdf 
Furrer, C. J., Skinner, E. A. y Pitzer, J. R. (2014). The influence of teacher and peer relationships on students' classroom engagement and everyday resilience. En D. J. Shernoff y J. Bempechat (Eds.), Engaging youth in schools: Empirically-based models to guide future innovations (pp. 101-123). Teachers College Press.

González, A., Paoloni, P. V., Donolo, D. y Rinaudo, C. (2015). Compromiso conductual y desafección con las actividades escolares: Explorando un modelo de facilitadores motivacionales. Anales de Psicología, 31(3), 869-878. https://doi.org/10.6018/analesps.32.1.176981

González-Alba, B., Cortés-González, P. y Rivas-Flores, J. I. (2020). Experiencia escolar, diversidad y ciudadanía justa. Un estudio biográfico-narrativo. Revista Internacional de Educación para la Justicia Social, 9(1), 41-58. https://doi.org/10.15366/riejs2020.9.1.002

Griffiths, A. J., Sharkey, J. D. y Furlong, M. J. (2009). Student engagement and positive school adaptation. En R. Gilman, E. S. Huebner y M. J. Furlong (Eds.), Handbook of positive psychology in schools (p. 197-211). Routledge.

Gutiérrez, M., Sancho, P., Galiana, L. y Tomás, J. M. (2018). Autonomy support, psychological needs satisfaction, school engagement and academic success: A mediation model. Universitas Psychologica, 17(5), 1-12. https://doi.org/10.11144/Javeriana.upsy17-5.aspn

Henson, R. K. y Roberts, J. K. (2006). Use of exploratory factor analysis in published research: Common errors and some comment on improved practice. Educational and Psychological Measurement, 66(3), 393-416. https://doi.org/10.1177/0013164405282485

Immekus, J. C. y Ingle, W. K. (2019). A test and cross-validation of the factor structure of the engagement versus disaffection with learning instrument among middle school students. Journal of Psychoeducational Assessment, 37(4), 504-511. https://doi.org/10.1177/0734282917742569

Institute for Research and Reform in Education. (1998). Research Assessment Package for Schools (RAPS): Manual for elementary and middle school assessments. Institute for Research and Reform in Education.

Jang, H., Kim, E. J. y Reeve, J. (2016). Why students become more engaged or more disengaged during the semester: A self-determination theory dual-process model. Learning and Instruction, 43, 27-38. https://doi.org/10.1016/j.learninstruc.2016.01.002

Kong, Q., Wong, N. y Lam, C. (2003). Student engagement in mathematics: Development of instrument and validation of construct. Mathematics Education Research Journal, 15(1), 4-21.

Kyriazos, T. A. (2018). Applied psychometrics: sample size and sample power considerations in factor analysis (EFA, CFA) and SEM in general. Psychology, 9(8), 86856.

https://doi.org/10.4236/psych.2018.98126

Lara, L., Saracostti, M., Navarro, J. J., de-Toro, X., Miranda-Zapata, E., Trigger, J. M. y Fuster, J. (2018). Compromiso escolar: Desarrollo y validación de un instrumento. Revista Mexicana de Psicología, 35(1), 52-62.

Lei, H., Cui, Y. y Zhou, W. (2018). Relationships between student engagement and academic achievement: A meta-analysis. Social Behavior and Personality: An International Journal, 46(3), 517-528.

https://doi.org/10.2224/sbp.7054

Lloret-Segura, S., Ferreres-Traver, A., Hernández- Baeza, A. y Tomás-Marco, I. (2014). El análisis factorial exploratorio de los ítems: una guía práctica, revisada y actualizada. Anales de Psicología, 30(3), 1151-1169.

https://doi.org/10.6018/analesps.30.3.199361

Lorenzo-Seva, U. y Ferrando, P. J. (2019). Factor analysis (Versión 10.10.01).

https://psico.fcep.urv.cat/utilitats/factor

Mahatmya, D., Lohman, B. J., Matjasko, J. L. y Farb, A. F. (2012). Engagement across developmental periods. En S. L. Christenson, A. L. Reschly y C. Wylie (Eds.), Handbook of research on student engagement (pp. 45-63). Springer. https://doi.org/10.1007/978-1-4614-2018-7_5

Martin, A. J. (2009). Motivation and engagement across the academic life span: A developmental construct validity study of elementary school, high school, and university/college students. Educational and Psychological Measurement, 69(5), 794-824. https://doi.org/10.1177/0013164409332214 
McHugh, R. K. y Otto, M. W. (2012). Refining the measurement of distress Intolerance. Behavior Therapy, 43(3), 641651. https://doi.org/10.1016/j.beth.2011.12.001

Ministerio de Educación y Deportes. (2017). Principales cifras del sistema educativo nacional. http://www.bnm.me.gov.ar/gigal/documentos/EL005678.pdf.

Miñano, P., Gilar, R. y Castejón, J. L. (2012). A structural model of cognitive motivational variables as explanatory factors of academic achievement in Spanish language and mathematics. Anales de Psicología, 28, $45-54$.

Miranda-Zapata, E., Lara, L., Navarro, J. J., Saracostti, M. y de-Toro, X. (2018). Modeling the effect of school engagement on attendance to classes and school performance. Revista de Psicodidáctica, 23(2), 102-109.

https://doi.org/10.1016/j.psicod.2018.02.003

Miserandino, M. (1996). Children who do well in school: Individual differences in perceived competence and autonomy in above-average children. Journal of Educational Psychology, 88, 203-214.

https://doi.org/10.1037/0022-0663.88.2.203

Molinari, A., y Sánchez Rosas, J. (2018). Compromiso comportamental: Propuestas para la evaluación de participación social académica. Contextos de Educación, 20(25), 90-100.

Montero, I. y León, O. G. (2002). Clasificación y descripción de las metodologías de investigación en Psicología. International Journal of Clinical and Health Psychology, 2(3), 503-508.

Peña, G., Cañoto, Y. y Angelucci, L. (2017). Involucramiento académico: una escala. Páginas de Educación, 10(1), 114136. https://doi.org/10.22235/pe.v10i1.1361

Reeve, J. y Shin, S. H. (2020). How teachers can support students' agentic engagement. Theory into Practice, 59(2), 150-161. https://doi.org/10.1080/00405841.2019.1702451

Reich, J., Thompson, M. G., Cowling, B. J., Iuliano, A. D., Greene, C., Chen, Y., Phadnis, R., Leung, N. H. L., Song, Y., Fang, V. J., Xu, C., Dai, Q., Zhang, J., Zhang, H. y Havers, F. (2020). Comparison of alternative full and brief versions of functional status scales among older adults in China. PloS One, 15(8), e0234698. https://doi.org/10.1371/journal.pone.0234698

Reschly, A. L. y Christenson, S. L. (2012). Jingle, jangle, and conceptual haziness: evolution and future directions of the engagement construct. En S. L. Christenson, A. L. Reschly y C. Wylie (Eds.), Handbook of research on student engagement (pp. 3-19). Springer. https://doi.org/10.1007/978-1-4614-2018-7_5

Rigo, D. Y. y Donolo, D. (2014). Entre pupitres y pizarrones. Retos en educación primaria: El aprendizaje con compromiso. Educatio Siglo XXI, 32(2), 59-80. https://doi.org/10.6018/j/202161

Sandoval-Muñoz, M. J., Mayorga-Muñoz, C. J., Elgueta-Sepúlveda, H. E., Soto-Higuera, A. I., Viveros-Lopomo, J. y Riquelme-Sandoval, S. V. (2018). Compromiso y motivación escolar: una discusión conceptual. Revista Educación, 42(2), 66-79. https://doi.org/10.15517/revedu.v42i2.23471

Sinatra, G. M., Heddy, B. C. y Lombardi, D. (2015). The challenges of defining and measuring student engagement in science. Educational Psychologist, 50(1), 1-13. https://doi.org/10.1080/00461520.2014.1002924

Sinclair, M. F., Christenson, S. L., Lehr, C. A. y Anderson, A. R. (2003). Facilitating student engagement: Lessons learned from Check \& Connect longitudinal studies. The California School Psychologist, 8(1), 29-41. https://doi.org/10.1007/BF03340894

Skinner, E. A., Kindermann, T. A. y Furrer, C. J. (2009). A motivational perspective on engagement and disaffection: Conceptualization and assessment of children's behavioral and emotional participation in academic activities in the classroom. Educational and Psychological Measurement, 69(3), 493-525. https://doi.org/10.1177/0013164408323233

Skinner, E., Furrer, C., Marchand, G. y Kindermann, T. (2008). Engagement and disaffection in the classroom: Part of a larger motivational dynamic? Journal of Educational Psychology, 100(4), 765-781.

https://doi.org/10.1037/a0012840 
Tian, L., Tian, Q. y Huebner, E. S. (2016). School-related social support and adolescents' school-related subjective well-being: The mediating role of basic psychological needs satisfaction at school. Social Indicators Research, 128(1), 105-129. https://doi.org/10.1007/s11205-015-1021-7

Timmerman, M. E. y Lorenzo-Seva, U. (2011). Dimensionality assessment of ordered polytomous items with parallel analysis. Psychological Methods, 16(2), 209-220. https://doi.org/10.1037/a0023353

Tinio, M. F. (2009). Academic engagement scale for grade school students. The Assessment Handbook, 2(1), 64-75.

Tomás, J. M., Gutiérrez, M., Sancho, P., Chireac, S. M. y Romero, I. (2016). El compromiso escolar (school engagement) de los adolescentes: Medida de sus dimensiones. Enseñanza \& Teaching, 34(1), 119-135.

https://doi.org/10.14201/et2016341119135

Veiga, F. (2013). Envolvimento dos alunos na escola: Elaboração de uma nova escala de avaliação. International Journal of Developmental and Educational Psychology, 1(1), 441-450.

Vernucci, S., Canet-Juric, L., Andrés, M. L. y Burín, D. (2017). Comprensión lectora y cálculo matemático: El rol de la memoria de trabajo en niños de edad escolar. Psykhe, 26(2), 1-13.

https://doi.org/10.7764/psykhe.26.2.1047

Vilà, R., Aneas, A., Rubio, M. J. y Freixa, M. (2019). El valor del compromiso del estudiante para la garantía de calidad de la educación superior. Alejándose del enfoque regulatorio. REICE. Revista Iberoamericana sobre Calidad, Eficacia y Cambio en Educación, 17(2), 107-122.

https://doi.org/10.15366/reice2019.17.2.006

Wang, M. T. y Hofkens, T. L. (2019). Beyond classroom academics: A school-wide and multi-contextual perspective on student engagement in school. Adolescent Research Review, 5, 1-15.

https://doi.org/10.1007/s40894-019-00115-z

Wang, M. T., Willet, J. B. y Eccles, J. S. (2011). The assessment of school engagement: Examining dimensionality and measurement invariance by gender and race/ethnicity. Journal of School Psychology, 49(4), 465-480. https://doi.org/10.1016/j.jsp.2011.04.001

Wieland, A., Durach, C. F., Kembro, J. y Treiblmaier, H. (2017). Statistical and judgmental criteria for scale purification. Supply Chain Management, 22(4), 321-328. https://doi.org/10.1108/SCM-07-2016-0230

Wilkinson, G. (1993). Wide range achievement test 3. Wide Range, Inc.

Wonglorsaichon, B., Wongwanich, S. y Wiratchai, N. (2014). The influence of students school engagement on learning achievement: A structural equation modeling analysis. Procedia-Social and Behavioral Sciences, 116, 17481755. https://doi.org/10.1016/j.sbspro.2014.01.467

World Medical Association (2013). Declaration of Helsinki - Ethical principles for medical research involving human subjects. https://www.wma.net/policies-post/wma-declaration-of-helsinki-ethical-principles-for-medical-research-involving-human-subjects/

Zamora, E., del Valle, M., Galli, J. I., Vernucci, S., Canet, Juric, L. y Richard's, M. (2020). Evaluación de la impulsividad por múltiples informantes: Escala de impulsividad de dominio específico en niños. Evaluar, 20(2), 35-50. 


\section{Anexo I}

\section{Cuestionario de Dimensiones y Facilitadores del Compromiso Escolar}

Por favor, leé cada una de las siguientes oraciones. Después, respondé qué tan de acuerdo estás vos con cada una de ellas, marcando con una cruz solamente la opción que corresponda. No hay respuestas correctas o incorrectas.

\begin{tabular}{lcccc}
\hline Muy de & $\begin{array}{c}\text { Algo de } \\
\text { acuerdo }\end{array}$ & $\begin{array}{c}\text { No muy de } \\
\text { acuerdo }\end{array}$ & $\begin{array}{c}\text { Para nada de } \\
\text { acuerdo }\end{array}$ & acuerdo \\
\hline
\end{tabular}

1. Me esfuerzo para que me vaya bien en la escuela.

2. Cuando estoy en clase, me siento bien.

3. Disfruto de aprender cosas nuevas en clase.

4. Me interesan las cosas que hacemos en clase.

5. En clase, pongo todo mi esfuerzo.

6. Me siento mal cuando estoy en clase.

7. Cuando estoy en clase, escucho atentamente.

8. Me aburro cuando mi maestro o maestra explica algo nuevo.

9. Me engancho con las actividades que hacemos en clase.

10. En la clase hago lo mínimo, como para zafar.

11. Me siento enojado cuando tengo que hacer actividades en clase.

12. Estar en clase es divertido.

13. No puedo hacer que me vaya bien en la escuela.

14. El mejor modo de sacarme buenas notas es caerle bien a mi maestro o maestra.

15. Hago la tarea en casa porque quiero entender el tema.

16. Trabajo en las actividades de clase porque me resultan interesantes.

17. Pienso que es importante hacer las actividades de clase.

18. Hago las actividades de clase porque quiero aprender cosas nuevas.

19. Siento que mi maestro o maestra me ignora.

20. Esforzarme es el mejor modo de que me vaya bien en la escuela.

21. No me va a ir bien en la escuela si a mi maestro o maestra le caigo mal.

22. Hago las actividades de clase porque es divertido.

23. Tengo que tener suerte para que me vaya bien en la escuela.

24. Hago la tarea en casa porque me gusta hacerla.

25. Me siento enojado cuando estoy con $\mathrm{mi}$ maestro o maestra. 
26. Hago las actividades de clase porque me importa que me vaya bien en la escuela.

27. No sé qué tengo que hacer para sacarme buenas notas.

28. Hago la tarea en casa porque quiero aprender cosas nuevas.

29. Puedo esforzarme mucho en la escuela.

30. No me siento feliz cuando estoy con mi maestro o maestra.

31. Hago la tarea en casa porque es divertido.

32. No tengo suerte en la escuela.

\section{Breve CV de los/as autores/as}

\section{Rosario Gelpi Trudo}

Licenciada en Psicología por la Universidad Nacional de Mar del Plata. Desarrolló su tesis de grado en el marco de un proyecto de evaluación de la autorregulación cognitiva y emocional y el rendimiento académico en población infantil. Fue becaria de la categoría Estudiante Avanzado de la Universidad Nacional de Mar del Plata, y adscripta a la docencia en las cátedras Introducción a la Psicología y Psicología Cognitiva de la Facultad de Psicología UNMdP. Actualmente se desempeña como Becaria Doctoral del CONICET en el Instituto de Psicología Básica, Aplicada y Tecnología - IPSIBAT-, llevando adelante un proyecto para conocer la influencia del uso de nuevas tecnologías en las funciones ejecutivas, el autocontrol y el desempeño académico en población infantil. Email: rosariogelpitrudo@gmail.com

ORCID ID: https://orcid.org/0000-0002-7201-6832

\section{Macarena Verónica del Valle}

Licenciada en Psicología por la Universidad Nacional de Mar del Plata. También realizó una Especialización en Psicoterapia Cognitiva en la Fundación Aiglé y un Master en Investigación en Educación en la Universidad de Valladolid, España. Actualmente, es estudiante del Doctorado en Psicología de la UNMDP y Becaria Doctoral del CONICET. Es docente en la cátedra de Teorías del Aprendizaje de la Universidad Nacional de Mar del Plata y auxiliar en proyectos de investigación del Instituto de Psicología Básica, Aplicada y Tecnología -IPSIBAT-. Macarena ha asistido a diversos cursos de posgrado y ha sido expositora en congresos de alcance nacional e internacional. Posee diversas publicaciones sobre procesos básicos, aprendizaje, funciones ejecutivas, características psicológicas, psicología cognitiva y metodología de investigación, medición y evaluación. Se área de trabajo son los procesos de autorregulación cognitiva y emocional en el ámbito educativo. Email: mdelvalle1989@gmail.com

ORCID ID: https://orcid.org/0000-0003-3549-7224

\section{Carolina Magalí Pagano}

Estudiante de la Licenciatura en Psicología en la Universidad Nacional de Mar del Plata. Desarrolló su tesis de grado en el marco de un proyecto de evaluación de autorregulación cognitiva y emocional y rendimiento académico en población infantil, participando del equipo de investigación en la toma de datos y en el análisis e interpretación de resultados. Realizó pasantías en el ámbito de la Psicología Educacional, asistiendo en equipos de orientación interdisciplinarios de dos colegios de la ciudad de Mar del Plata. También se desempeña en el ámbito del acompañamiento terapéutico infantil. Email:carolinamagali64@gmail.com

ORCID ID: http://orcid.org/0000-0002-9834-0964 


\section{María Laura Andrés}

Licenciada y Doctora en Psicología por la Universidad Nacional de Mar del Plata. También es Master en Psicología, Educación y Desarrollo por la Universidad de Cádiz, España y Magíster en Psicología Cognitiva y Aprendizaje por FLACSO-UAM (Facultad Latinoamericana de Ciencias Sociales, Argentina; Universidad Autónoma de Madrid, España). Es Investigadora asistente del CONICET y docente de la cátedra Psicología Cognitiva de la Facultad de Psicología de la Universidad Nacional de Mar del Plata. Trabaja actualmente en el Instituto de Psicología Básica, Aplicada y Tecnología. Ha realizado investigaciones en las temáticas de regulación emocional, salud mental y desempeño académico. Actualmente trabaja en la puesta a prueba de un modelo teórico para conocer las relaciones de las funciones ejecutivas y la tolerancia al distrés sobre el desempeño académico en niños y coordina el desarrollo de un programa de aprendizaje socioemocional para el aula. Email: marialauraandres@gmail.com

ORCID ID: https://orcid.org/0000-0003-4398-2043

\section{Ana García Coni}

Licenciada y Doctora en Psicología por la Universidad Nacional de Mar del Plata. Es investigadora asistente del CONICET, y docente de la cátedra Teorías del Aprendizaje de la Facultad de Psicología de la Universidad Nacional de Mar del Plata. Trabaja actualmente en el Instituto de Psicología Básica, Aplicada y Tecnología. Su lugar de trabajo es el Instituto de Psicología Básica, Aplicada y Tecnología (IPSIBAT; UNMdP - CONICET). Su área de trabajo es la Psicología Cognitiva, la Psicología del Desarrollo y la Psicología Educacional, específicamente la flexibilidad cognitiva, la categorización y los procesos inhibitorios en niños escolares y adolescentes. También trabaja sobre el impacto que las creencias implícitas sobre la inteligencia tienen en el desempeño escolar. Email: anagconi@gmail.com

ORCID ID: https://orcid.org/0000-0002-6304-7880

\section{Lorena Canet Juric}

Doctora en Psicología y Mg. en Psicología Cognitiva y Aprendizaje por FLACSO-Universidad Autónoma de Madrid. Investigadora CONICET. Es docente de Psicología Cognitiva de la Facultad de Psicología de la Universidad Nacional de Mar del Plata. Trabaja actualmente en el Instituto de Psicología Básica, Aplicada y Tecnología. Posee numerosas publicaciones en revistas científicas sobre temas vinculados al aprendizaje y las Funciones Ejecutivas. Ha coordinado y/o participado en numerosas actividades de extensión destinadas a docentes y a padres. Su tema de investigación actual es la exploración de las relaciones entre diversas herramientas de autorregulación (memoria de trabajo e inhibición) y su vinculación con el autocontrol y el rendimiento escolar en los niños. Email: lcanetjuric@gmail.com

ORCID ID: https://orcid.org/0000-0003-4147-4889 